\title{
IR and Masing Properties of Bright IRAS Sources
}

\section{B. M. Lewis}

\author{
Arecibo Observatory, Puerto Rico
}

Comparisons between color-color diagrams of OH/IR stars with/out IR flux and/or galactic position constraints show that little of the color dispersion is innate: the most precise colors come from high flux samples away from the Galactic Center. It is advantageous to delineate the color range of O-rich shells by only color selecting objects in the IRAS Point Source Catalog (PSC) with S(25) > 100 Jy, which yields 333 objects. All but 2 of these have published LRS spectra, which often suffice to determine whether an object is carbon or oxygen rich. About $80 \%$ of them have been searched for $1612 \mathrm{MHz}$ masers, and $\approx 70 \%$ for $\mathrm{SiO}$, water and/or mainline $\mathrm{OH}$ masers. As bright objects, most "oddballs" have been investigated at other wavelengths too. This data, together with information gleaned from a SIMBAD search, was then used to distinguish emission-line, $T$ Tauri and carbon stars from the predominent residuum of $\mathrm{O}$-rich shells.

The O-rich shells with masers fall in a well defined sequence in an IR color-color diagram. The precise IR colors of this sample show that OH/IR stars normally have (2512) $\mu \mathrm{m}<+0.1$, while proto planetary nebulae are generally redder. Most O-rich shells with (25-12) $\mu \mathrm{m}<-0.5$ exhibit masers (water, $\mathrm{SiO}$, and/or mainline $\mathrm{OH}$ ), while those with $1612 \mathrm{MHz}$ masers are also those with (25-12) $\mu \mathrm{m}>-0.5$. Nevertheless this process identifies 17 red O-rich shells without $1612 \mathrm{MHz}$ masers, in addition to 10 with featureless LRS spectra that may perhaps be red carbon stars. In general $16 \%$ of the O-rich sample at all colors are without detected masers, while up to $75 \%$ of all red color-selected sources have $1612 \mathrm{MHz}$ masers.

There are several objects with anomalous $60 \mu \mathrm{m}$ colors: some are normal seeming $\mathrm{OH} / \mathrm{IR}$ stars (R Aqr, UX Cyg, GX Mon); one is a carbon star (IRAS 18288-0837); while others have masers but not $1612 \mathrm{MHz}$ masers (Iras 11385-5517, IRAS 17163-3835, IRAS 18135-1641) and several are still to be checked (IRAS 18389-0424, IRAS 18457-0154, IRAS 20312+4035). In most of these instances the IRAS $60 \mu \mathrm{m}$ image shows a confusing source nearby, which has perturbed the listed flux: almost all have IRAS flux quality flags set. These examples of grave color perturbations occur even handedly in all subsets: their existence is indicative of problems in extracting reliable fluxes in $\approx 3 \%$ of cases. There appear to be no O-rich objects whose intrinsic colors place them well above the sequence of circumstellar shells.

A five parameter relation was fitted to the set of objects with detected masers that are not deemed to be PPN. This fit assumes that observational errors are entirely in the measured (60-25) $\mu \mathrm{m}$ color, whereon $\langle\Delta(60-25) \mu \mathrm{m}\rangle=0.005 \pm 0.011(\sigma=0.143)$. If the minimum two-dimensional residual for each object from the same relation is computed, $<$ res $>=0.005 \pm 0.007(\sigma=0.100)$. These $\sigma$ are consistent with the advertised precision of IRAS fluxes, which suggests that the whole of the scatter in colors of O-rich shells can plausably be attributed to imprecision in their colors. 